\title{
Genome-wide characterization and evolutionary analysis of heat shock transcription factors (HSFs) to reveal their potential role under abiotic stresses in radish (Raphanus sativus L.)
}

\author{
Mingjia Tang, Liang Xu, Yan Wang, Wanwan Cheng, Xiaobo Luo, Yang Xie, Lianxue Fan and Liwang Liu* (i)
}

\begin{abstract}
Background: Abiotic stresses due to climate change pose a great threat to crop production. Heat shock transcription factors (HSFs) are vital regulators that play key roles in protecting plants against various abiotic stresses. Therefore, the identification and characterization of HSFs is imperative to dissect the mechanism responsible for plant stress responses. Although the HSF gene family has been extensively studied in several plant species, its characterization, evolutionary history and expression patterns in the radish (Raphanus sativus L.) remain limited.

Results: In this study, 33 RsHSF genes were obtained from the radish genome, which were classified into three main groups based on HSF protein domain structure. Chromosomal localization analysis revealed that 28 of 33 RsHSF genes were located on nine chromosomes, and 10 duplicated RsHSF genes were grouped into eight gene pairs by whole genome duplication (WGD). Moreover, there were 23 or 9 pairs of orthologous HSFs were identified between radish and Arabidopsis or rice, respectively. Comparative analysis revealed a close relationship among radish, Chinese cabbage and Arabidopsis. RNA-seq data showed that eight RsHSF genes including RsHSF-03, were highly expressed in the leaf, root, cortex, cambium and xylem, indicating that these genes might be involved in plant growth and development. Further, quantitative real-time polymerase chain reaction (RT-qPCR) indicated that the expression patterns of 12 RsHSF genes varied upon exposure to different abiotic stresses including heat, salt, and heavy metals. These results indicated that the RsHSFs may be involved in abiotic stress response.

Conclusions: These results could provide fundamental insights into the characteristics and evolution of the HSF family and facilitate further dissection of the molecular mechanism responsible for radish abiotic stress responses.

Keywords: Abiotic stress, Heat shock transcription factors (HSFs), Radish, Reverse-transcription quantitative PCR (RT-qPCR)
\end{abstract}

\footnotetext{
* Correspondence: nauliulw@njau.edu.cn

National Key Laboratory of Crop Genetics and Germplasm Enhancement, Key

Laboratory of Horticultural Crop Biology and Genetic Improvement (East

China) of MOA, College of Horticulture, Nanjing Agricultural University,

Nanjing 210095, People's Republic of China
}

(c) The Author(s). 2019 Open Access This article is distributed under the terms of the Creative Commons Attribution 4.0 International License (http://creativecommons.org/licenses/by/4.0/), which permits unrestricted use, distribution, and reproduction in any medium, provided you give appropriate credit to the original author(s) and the source, provide a link to the Creative Commons license, and indicate if changes were made. The Creative Commons Public Domain Dedication waiver (http://creativecommons.org/publicdomain/zero/1.0/) applies to the data made available in this article, unless otherwise stated. 


\section{Background}

Many inevitable environmental factors (e.g., heat, drought, flooding, salinity and heavy metals) trigger abiotic stress, affect plant growth and consequently increase crop losses [1-3]. Heat stress (HS), one of the major abiotic stresses, severely inhibits crop growth and development. These effects have had devastating economic impacts on the yield and quality of rice, wheat, maize and vegetable crops [4-6]. Heat shock transcription factors (HSFs) are important regulators that could contribute to controlling the differential expression of heat shock proteins (HSPs) and other functional genes in the process of protecting plants from heat and other stresses including chilling, salinity, drought and heavy metal $[7,8]$. It is imperative to clarify the molecular mechanisms that govern how plants respond and adapt to HS.

Plants have developed multiple defense mechanisms and strategies to cope with adverse conditions and respond accordingly [9-11]. Under abiotic stresses, the induction of myriad proteins, including transcription factors (TFs), could regulate the expression of specific functional genes to enhance plant resistance through signal transduction pathways. Reactive oxygen species (ROS)-scavenging enzymes and HSPs are important functional proteins induced by $\mathrm{HS}$, and their corresponding genes are targets of several HS-responsive TFs [12-14]. Previous studies indicate that $H S F A 6 b$ vitally acts as a downstream regulator of the ABA-mediated heat stress response (HSR) in Arabidopsis thaliana [15]. Additionally, in the absence of HS, HSP genes expression is induced by the overexpression of constitutively active HsfA1d without temperature-dependent repression (TDR) domain, thus conferring effective thermotolerance in Arabidopsis [16]. In the tomato, cadmium (Cd) tolerance is conferred by HsfAla through activating COMT1 gene transcription, which partially upregulates HSP expression by inducing melatonin in accumulation [8].

In plants, the HSFs have a conserved modular structure with considerable size and sequence variability [9, 17-19]. For example, all HSFs have an N-terminal DNA-binding domain (DBD) and an adjacent bipartite oligomerization domain (HR-A/B), while only a proportion contain a nuclear localization signal (NLS) domain, nuclear export signal (NES) domain and C-terminal activator domain (CTAD) [9]. According to the peculiarities of the adjacent hydrophobic amino acid residues (HR-A/ B), plant HSFs can be divided into three types, class A, B and $C$ [20-22]. Numerous HSF families have been isolated from a range of plant species, including Chinese cabbage (Brassica.rapa ssp. pekinensis) [23], Arabidopsis [24], carrot (Daucus carota) [13], peanut (Arachis hypogaea) [7], rice (Oryza sativa) [25], maize (Zea mays) [26], Chinese white pear (Pyrus bretschneideri) [27], poplar (Populus trichocarpa) and barrelclover (Medicago truncatula) [28]. However, the comprehensive characteristics of HSF genes remains unknown in root vegetable crops, including radish.

Radish (Raphanus sativus L., $2 \mathrm{n}=18$ ), belonging to the Brassicaceae family, is one of the most economically important annual or biennial root vegetable crops [29]. Previous studies showed that 26 known and 19 novel microRNAs (miRNAs) are differentially expressed under HS in radish roots [4]. Moreover, there are several differentially expressed genes (DEGs) involved in heat stress response process in radish [30]. Recently, the available radish genome sequence provided a useful resource for whole-genome identification of TF families [31]. Nevertheless, little information on systematic characterization of HSF genes and their families in response to abiotic stresses is available in radish. This study aimed to to isolate the full-length RsHSF sequences from the radish genome, map the RsHSFs onto chromosomes and explore their expression in response to abiotic stresses. Moreover, RsHSF orthologs and paralogs were obtained and the expression pattern of RsHSFs in different radish tissues was investigated. The outcomes of this study provide an opportunity to further explore the roles of HSF genes involved in abiotic stresses and present the expansion and evolutionary history of the HSF gene family in radish.

\section{Results}

Identification and characterization of radish HSF proteins

To comprehensively identify the candidate HSF proteins in radish, a profile Hidden Markov Model (HMM) [32] search against NODAI radish genome protein sequences with the HSF domain (Pfam: PF00447) was performed. A total of 55 putative RsHSF gene sequences were obtained from the whole genome. SMART (http://smart.embl-heidelberg.de/) and Pfam (http://pfam.xfam.org/) were used to remove proteins with incomplete hsf-type DBD domain, while MARCOIL (http://toolkit.tuebingen.mpg. $\mathrm{de} /$ marcoil) was used to confirm OD (HR-A/B) domain presence. After removing sequences that encoded proteins without the complete DBD, OD (HR-A/B) and/or start/ stop codons, 33 RsHSF genes remained for further analysis (Additional file 1: Table S1-S3). Subsequently, the conserved domains of all retained genes were identified by Heatster. The DBD, which consists of approximately 100 amino acids (AAs) at the N-terminus, was the most conserved domain. Overall, 31 AAs of the DBD domain were highly conserved among all RsHSFs (Additional file 2: Figure S1). NLS and NES are crucial for HSF intracellular distribution between the nucleus and cytoplasm. All RsHSFs had an NES, whereas only 13 contained the NLS (Additional file 3: Table S4).

Furthermore, the physical and chemical properties of 33 RsHSF proteins were analyzed with ExPASy (Additional file 3: Table S4). Among these, the RsHSF protein 
sizes varied from 238 to 2427 AAs with molecular weights (MWs) from 27.80 to $274.05 \mathrm{KDa}$, respectively. Additionally, the theoretical pI of most RsHSF proteins was $<7$, with the exception RsHSF-04, RsHSF-05, RsHSF-06 and RsHSF-25. All RsHSFs were classified as unstable proteins according to the instability index. The aliphatic index may be a positive factor that increased globular protein thermostability, and RsHSF-25 had the largest aliphatic index (77.14). Most RsHSFs showed similar physical and chemical properties, while different AA sequences in non-conserved regions may alter some of the molecular characteristics.

\section{Intron-exon structure and conserved motif distribution}

To obtain information about the diversity of RsHSF gene structure, the full length of complementary DNA (cDNA) sequences were compared with the corresponding genomic DNA sequences through Gene Structure Display Server (GSDS). To further analyze RsHSF protein motif distribution, whole sequences were subjected to the MEME web server and a total of 25 AA motifs were generated (Additional file 2: Figure.S2).

Overall, 25 of 33 RsHSF genes had one intron, while the ramaining members have two or more introns. Additionally, proteins within the same subgroups had similar motif structures. All RsHSF proteins harbored motifs 1, 2 and 4, all of which highly correspond to the conserved DBD. Moreover, RsHSF-07, RsHSF-14 and RsHSF-26 in subgroup
A6 presented a similar gene and motif structure. However, some motifs were only detected in specific RsHSF protein classes. For example, motif 3 was identified in classes A and $\mathrm{C}$, while motifs 9 and 17 only existed in class A1. Intriguingly, the motifs 5 and 19 were only identified in class B. A proportion of RsHSF proteins in the same class exhibited similar motif distribution, indicating that these proteins might have conserved functions (Fig. 1).

\section{Comparative and phylogenetic analysis of RsHSF genes}

To investigate the distribution of HSF subfamilies and the evolutionary relationship among different species, 33 RsHSFs combined with 35 BrHSFs, 19 VvHSFs, 25 OsHSFs and 21 AtHSFs identified from Chinese cabbage (B. rapa ssp. pekinensis) [33], grape ( $V$. vinifera) (https:// phytozome.jgi.doe.gov/pz/portal.html) [34, 35], rice (O. sativa) (http://rice.plantbiology.msu.edu/) [36] and Arabidopsis (http://www.arabidopsis.org/) [37] were employed for comparative analysis (Additional file 4: Table S5). Based on the number of AA residues between the $\mathrm{A}$ and $B$ portions of the HR-A/B region, RsHSFs were classified into three classes, namely A, B and C (Fig. 1).

A phylogenetic tree was constructed using maximum likelihood methods by IQ-TREE [38] with the full sequences of the 133 HSF proteins consisted of 33 RsHSFs, 35 BrHSFs, 19 VvHSFs, 25 OsHSFs and 21 AtHSFs (Fig. 2). All proteins were categorised into three groups corresponding to the HSF classes A, B and C.

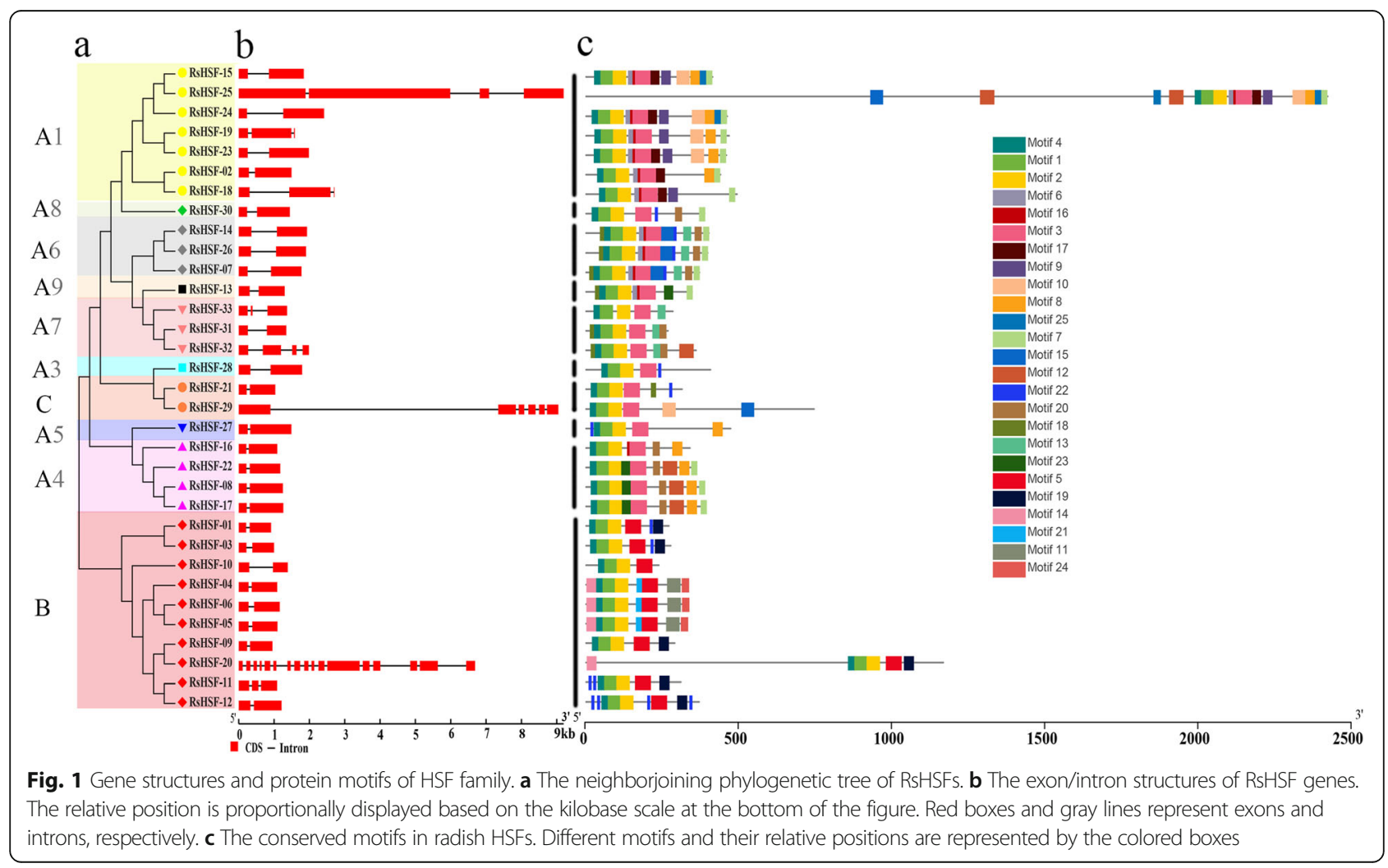




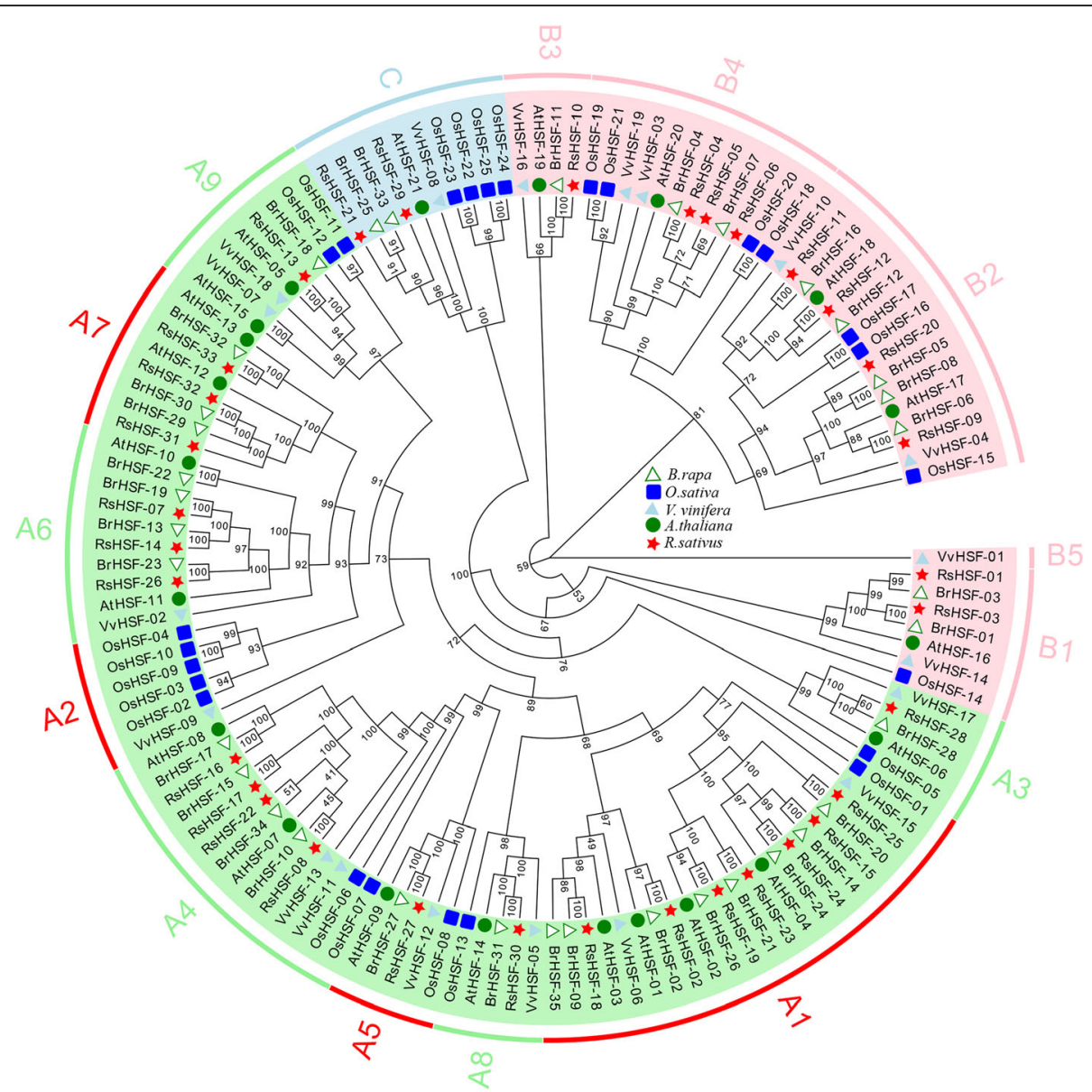

Fig. 2 Phylogenetic tree constructed using the maximum likelihood method by IQ-TREE, with HSF proteins in radish, Chinese cabbage, Arabidopsis, grape and rice. Bootstrap values were generated using UFBoot with 1000 replicates and super-fast bootstraps were interpreted as a true node if the support was above 0.95

Additionally, there were 9 RsHSF class A subgroups clearly obtained on the basis of the bootstrap values and phylogenetic analysis of Arabidopsis and rice. According to the phylogenetic tree, class $A$ consisted of 8 subclasses (A1, A3, A4, A5, A6, A7, A8, and A9) and contained the most RsHSF numbers, while class $C$ accounted for the least proportion of HSFs among these five species. Interestingly, no RsHSFs, AtHSFs or BrHSFs clustered into classes A2, whereas AcHSFs did not appear in classes A6 and A7. This finding suggests that most RsHSF genes are more closely related to their corresponding homologous genes in Chinese cabbage and Arabidopsis.

\section{Chromosomal location and duplication of RsHSF genes}

To obtain the chromosome distributions of RsHSF genes, their DNA sequences were physically plotted on the chromosomes through blast searches against the genomic sequences (Additional file 5: Table S6). In total, 28 RsHSF genes were mapped on the chromosomes with uneven distribution, with exception of five genes (RsHSF-22, 23,
25, 29 and 33) (Fig. 3). Chromosome (Chr.) 5 had the largest number of RsHSF genes among three classes, followed by Chr. 4 (5 RsHSF genes from class A and B). Only one RsHSF gene was present in Chr. 8, while Chr. 1, 6 and 9 harbored two genes. Moreover, all the class B RsHSF genes were mapped on the chromosomes.

Genome duplications have been recognized and contributed to the expansion of gene family in plants $[39,40]$. MCScanX was used to obtain information about origins of duplicate RsHSF genes in radish genome [41]. 13 (46.4\%) RsHSF genes were duplicated and retained from a whole genome duplication (WGD) event and $11(39.3 \%)$ were duplicated and retained from a singleton event (Additional file 6: Table S7). Eight WGD events of 10 duplicated RsHSF genes were identified and classified into four groups. Among these four groups, two harbored three genes (RsHSF-07, RsHSF-14 and RsHSF-26; RsHSF-08, RsHSF-16 and RsHSF-17), and these six genes were located on five chromosomes (Chr.2, 4, 5, 6 and 9). The other two groups contained 


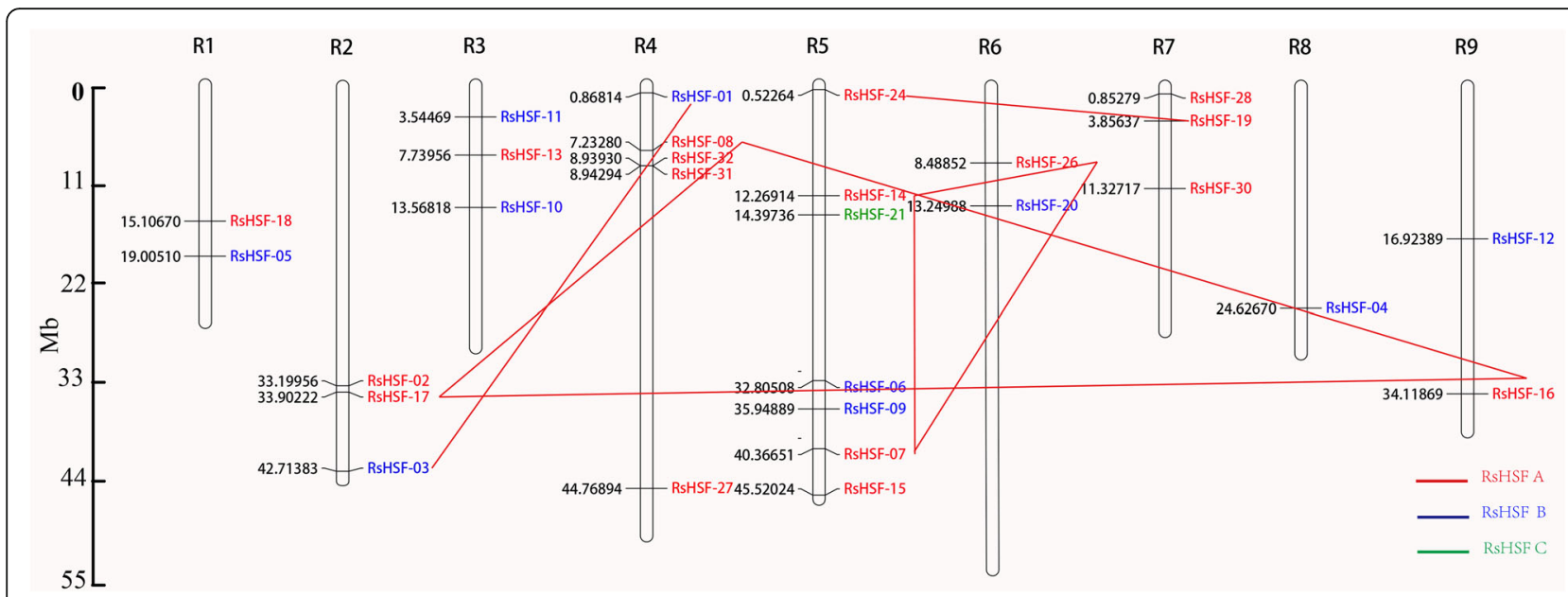

Fig. 3 Distribution of RsHSF genes on nine chromosomes. Red, blue and green word represent class A, B and C, respectively. Red lines connect the RsHSF duplicated genes

two WGD events of four genes, and one duplication event harbored RsHSF-01 and RSHSF-03 which were located on Chr. 2 and 4, respectively (Fig. 3). Only one WGD event (RsHSF-07and RsHSF-14) took place within the same chromosome (Fig. 3, Additional file 7: Table S8). These results revealed that WGD or segmental duplication played an important role in the expansion of the radish HSF gene family.

\section{Identification of orthologous and paralogous HSF genes}

Polyploidization events contributed significantly to the evolution of flowering plants [42]. Previous studies showed that the common ancestor of Brassica and Raphanus have experienced $\alpha$ ' whole-genome triplication (WGT) event since its divergence from Arabidopsis. However, the gene losses of orthologous groups between Arabidopsis, Brassica and Raphanus for protein-coding genes occurred in both the Brassica and Raphanus lineages $[39,42]$. Orthologous HSF genes among radish, Arabidopsis and rice were identified for triplication assessment using the Orthomcl-pipeline [43]. In total, there were 23 pairs of orthologous HSFs between radish and Arabidopsis, and eight paralogous gene pairs were identified in radish (Additional file 8: Table S9). It was reported that there are only six orthologous gene pairs between Arabidopsis and rice [23]. In addition, seven orthologous HSF gene pairs were identified between radish and rice (Additional file 8: Table S9). Among the orthologous HSF gene pairs between radish and Arabidopsis, AtHSF-11 and AtHSF-20 had three orthologous genes, three AtHSFs have two orthologous genes and 11 AtHSFs only had one orthologous gene (Additional file 2: Figure S3). These results indicate that many orthologous groups experienced gene losses. Moreover, five genes (RsHSF-01, RsHSF-12, RsHSF-13, RsHSF-20 and
RsHSF-32) had no orthologous gene in either Arabidopsis or rice. These results provide useful resource and reference for further exploring the functions of RsHSF genes in radish (Fig. 4).

\section{Expression pattern of RsHSF genes in different tissues}

To determine spatiotemporal expression patterns of RsHSF genes, the Reads Per Kilobase Per Million (RPKM) values of the 33 RsHSF genes in different tissues and developmental stages were collected from RNA-Seq data and presented in a heatmap (Fig. 5, Additional file 9: Table S10). In general, a proportion of RsHSF genes were not expressed in several tissues and developmental stages. As shown in Fig. 5, the RPKM value varied from 0 to 127.5 among the 33 RsHSFs genes. The number of RsHSF genes with RPKM $>1$ was 14 (42.4\%). It was found that the RPKM values of four RsHSFA1s (RsHSF-15, 19, 23, 25) were $<6$ during all tissues and stages. Notably, among the other three RsHSFA1 (RsHSF-02, 18, 24) genes, RsHSF-18 and RsHSF-24 were highly expressed in the cortex, cambium and xylem. The majority of RsHSF genes exhibited differential expression patterns in the various tissues and developmental stages. RsHSF-21 and RsHSF-29 (class C) were relatively lowly expressed during all developmental stages. In addition, RsHSF-13 expression increased significantly in root after 7 days after sowing (DAS), and it was increased in leaves after 7 and 40 DAS as well. RsHSF-30 (class A8) expression was higher compared to RsHSF-28 (class A3) during all developmental stages. Besides, RsHSFB genes exhibited stage-specific expression patterns. Among class B, both RsHSF-04 and RsHSF-10 were lowly expressed during all stages, while RsHSF-03 was highly expressed. 


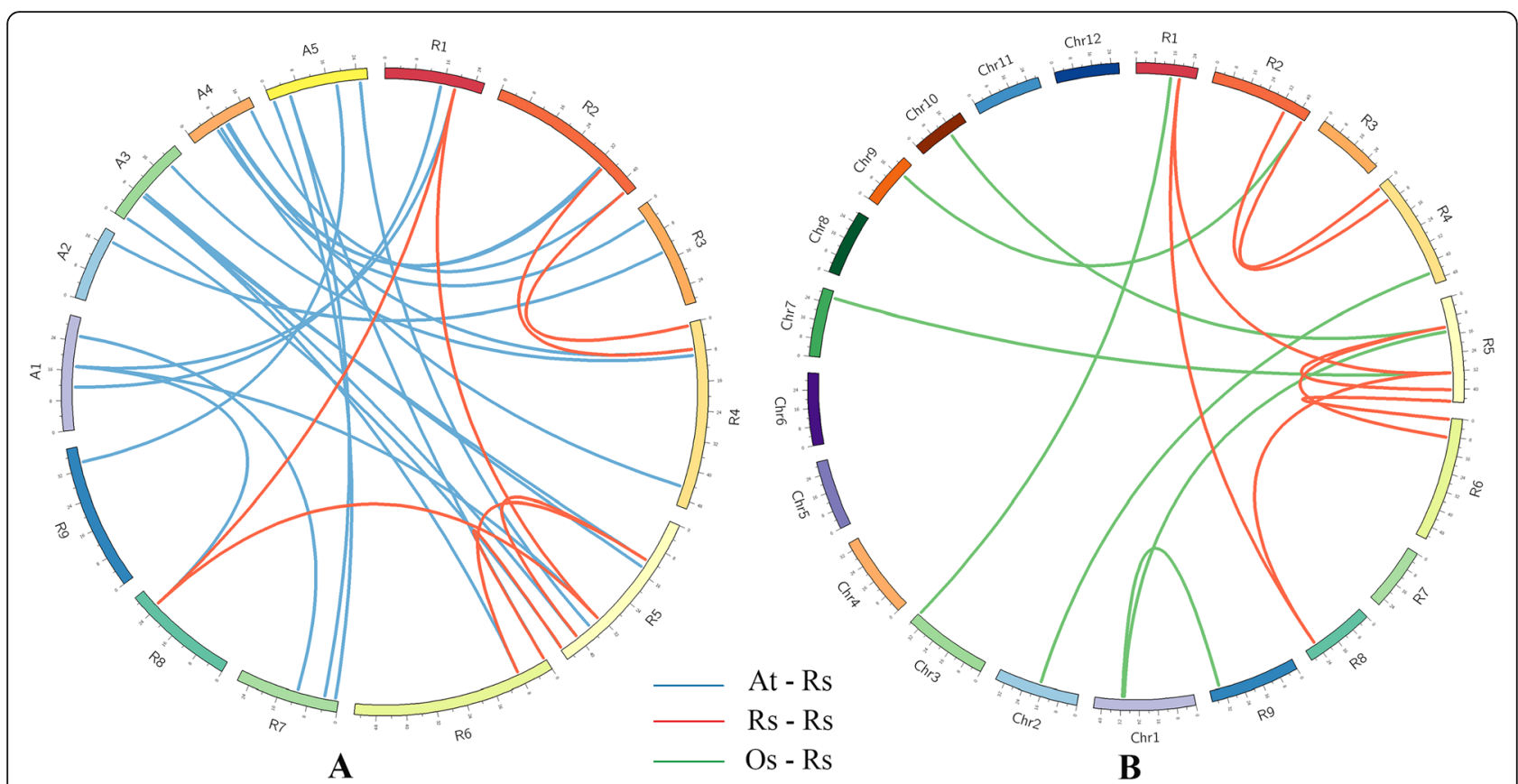

Fig. 4 Orthologous and paralogous genes of HSF. a Radish (R01-R09) and five Arabidopsis chromosome (A1-A5) maps were based on orthologous and paralogous pair positions; b Radish and rice chromosome (Chr1-Chr12) maps were based on orthologous and paralogous pair positions with Circos

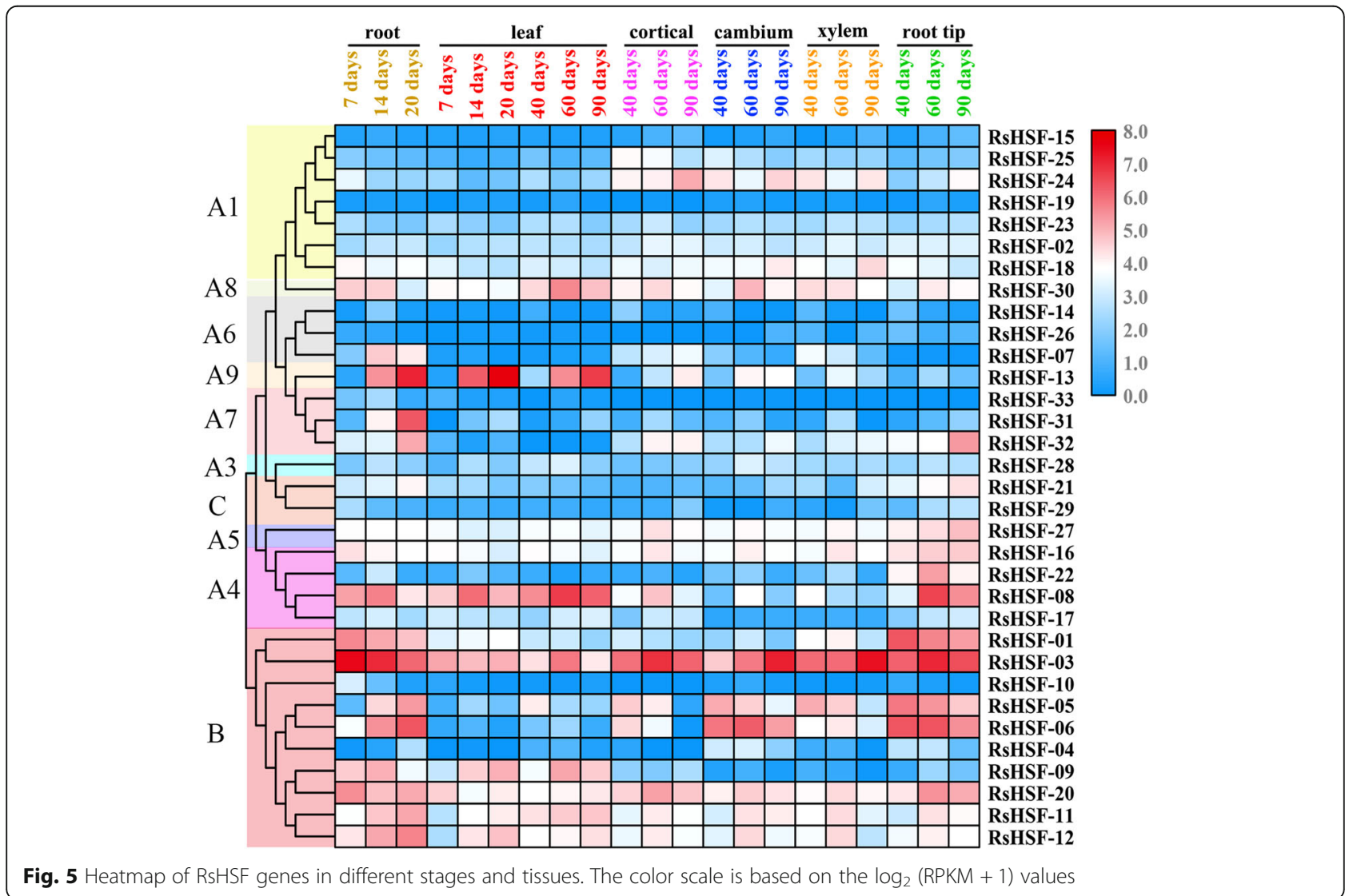


RsHSF gene expression profile under abiotic stresses

RNA-Seq data showed that some HSF genes were differentially expressed under disparate stresses. The transcription levels ( $\log _{2}$ (Fold Change) and $\log _{2}$ TPM value) of certain RsHSF genes from the NCBI SRA were further analyzed (Additional file 2: Figure S4). Several HSF genes, including HSFA1s were up-regulated in response to heat and salt stresses. RsHSFA4s (RsHSF-08, RsHSF-16 and RsHSF-22) were up-regulated under salt and lead $(\mathrm{Pb})$ stresses, while they were down-regulated under HS. Additionally, HSFC1 (RsHSF-21) expression increased after $\mathrm{Cd}$ and salt treatments. HSFA1s as master regulators play vital roles in the HSR and activation of transcriptional networks. Expression levels of genes that encode $\mathrm{HS}$ responsive TFs including $D R E B 2 A, H S F A 7 s$ and HSFBs, are directly regulated by HSFA1s [44]. HsfA4a is induced by oxidative stress, including $\mathrm{HS}$, and regulates $A P X 1$ expression [45]. To gain insight into the expression patterns of HSF genes in radish, 12 RsHSF genes that belonged to different groups were verified by $\mathrm{RT}$-qPCR analysis under heat, salt, Pb, and Cd stress (Additional file 10: Table S11). Overall, the gene expression patterns varied significantly under various treatments (Fig. 6). Most genes were upregulated after $24 \mathrm{~h}$ HS exposure, while some genes were down-regulated after HS for $6 \mathrm{~h}$ (Fig. 6), such as RsHSF16, RsHSF-22 and RsHSF-29. Moreover, RsHSF-18 expression was highly increased after $6 \mathrm{~h}$ HS, implying

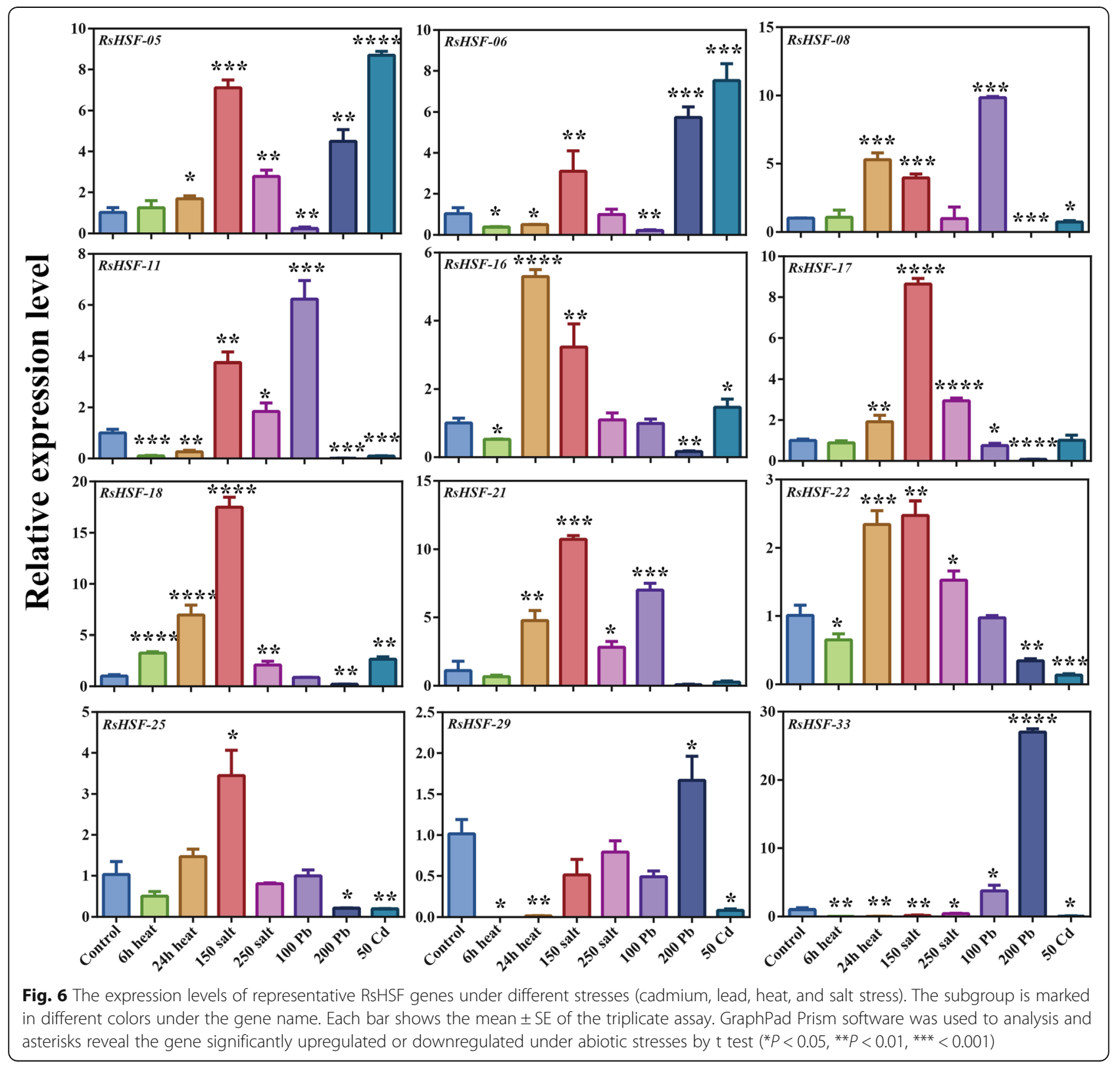


that it can quickly respond to this stress. In addition, RsHSF-18 was up-regulated under Cd treatment and during all heat and salt stress time points, while RsHSF33 was only up-regulated in response to $\mathrm{Pb}$ stress. RsHSF-05, RsHSF-06, and RsHSF-33 were up-regulated under $200 \mathrm{mg} / \mathrm{L} \mathrm{Pb}$ treatment and are likely involved in the $\mathrm{Pb}$ stress response. RsHSF-11 and RsHSF-22, exhibited relatively high expression level under salt stress, indicating that these two RsHSFs might play crucial roles in the biological process of salt stress response.

\section{Discussion}

Radish (Raphanus sativus L.), an ancient cultivated crop worldwide, is an important human dietary component. Industrial development, climate change and increased areas of contaminated soil considerably affect the expansion and healthy cultivation of the radish. The HSFs family allows plants to cope with abiotic stresses (e.g. heat, $\mathrm{Cd}$ and high light) by regulating gene expression to prevent damage $[15,46]$. Recently, the availability of increasingly complete genome sequencing has enabled the application of a bioinformatics approach to identify various gene families, including HSF in different species [27, 28]. However, the available information about the RsHSFs is limited. This study is the first comprehensive overview of the HSF gene family within the radish genome.

\section{Genome-wide identification of RsHSF genes}

It is widely accepted that the HSF-type DBD and OD domains are necessary HSF structural components. The highly conserved DBD is composed of a three-helical bundle ( $\mathrm{H} 1, \mathrm{H} 2$ and $\mathrm{H} 3)$ and a four-stranded antiparallel B-sheet [9]. The HR-A/B, connected to the DBD by a flexible linker of variable length (15-80 AAs), is comprised of hydrophobic heptad repeats $[47,48]$. In brief, the DBD ensures HSFs combine with HSE, and the OD is the basis for differentiating the three HSF classes. It is reported that the DNA-binding domain of plant HSF is separated by a single intron. Although the position of this intron is unanimous in all HSF DBDs, the intron size is variable [21]. Therefore, we predicted the conserved domain using Heatster online tools [49] after performing Pfam and MARCOIL analysis. Consequently, 33 RsHSF genes were identified through genome-wide analysis (Additional file 1: Table S1-S3).

Among five plant species, 133 HSFs divided into three major classes were used to analyze the relationships. The results suggested that HSFs originated prior to the divergence of these species. Phylogenetic analysis evidenced that RsHSFs were more similar to BrHSFs and AtHSFs than OsHSFs, which corresponds with the fact that the radish, Chinese cabbage and Arabidopsis belong to Brassicaceae family (Fig. 2). The radish HSF number (33) was larger than that in Arabidopsis (21) and grape (19). Previous studies showed that distinct plants species harbor different numbers of HSFs, and land plants have more TFs than algae. These results indicate that the number of HSFs is probably related to the evolution and the growth environment [13].

\section{The evolutionary characterization of the RsHSF family}

Gene duplication plays a major role in the evolution of novel gene functions and the expansion of gene families [36]. Among the evolutionary process in plants, gene duplication that increases the genome size and relaxes selection on one gene copy, is regarded as the primary driving force to allow the acquisition of new function, and enhance environmental adaptability $[13,50]$. In Brassicaceae family, there were three WGDs after an Arabidopsis lineage diverged from the monocot lineage. The most recent WGD event occurred 50-65 million years ago, which was earlier than the divergence of plants in the Brassicaceae family $[40,51,52]$. Many gene families are expanded in higher plants, including $C M L$ [53], ALDHs [54], MADS-Box [55], NAC [56] and bHLH [57]. Current evidence shows that the evolution patterns of $\alpha$ ' duplicates are similar in Raphanus and Brassica [40]. For RsHSFs evolution, the expansion was due to eight duplicated pairs, which are consistent with those in Chinese cabbage.

Polyploidization events apparently occurred 3-12 million years after independence of the Arabidopsis-Brassica lineages, and the time of the Raphanus genus diverging from Brassica was longer than previously predicted $[58,59]$. Furthermore, recent reports revealed that $\sim 60 \%$ of genes that belong to the neopolyploid ancestor of Raphanus and Brassica disappeared due to a WGT event. However, a considerable number of genes still exist within the Raphanus and Brassica genomes, a phenomenon that represents the benefit of this evolutionary novelty [40]. Phylogenetic analysis of HSF genes among radish, Arabidopsis and Chinese cabbage indicated that many RsHSF genes are highly similar to their corresponding Arabidopsis and Chinese cabbage homologs. Moreover, a large number of the identified RsHSFs were detected as orthologous genes in Arabidopsis, including RsHSF-04, RsHSF-05 and RsHSF-06, which were the orthologous genes of AtHSF-20 (Fig. 4). These results provide valuable cues on further understanding the functions of these highly homologous genes in radish. For instance, RsHSF-07, RsHSF-14 and RsHSF-26 were highly similar to AtHSF-11, which is vital for HS tolerance and is a downstream regulator during the ABA-mediated stress response [15]. In this study, we identified eight pairs of RsHSF paralogs that might be related to the extended genome triplication of the radish. Collectively, the RsHSF gene family expansion may be largely related 
with gene duplication, and the identification of the orthologous gene pairs between radish and Arabidopsis provided a reference for exploring the roles of HSFs under different stresses in radish.

\section{The potential roles of differentially expressed RsHSF genes}

HSFs play important roles in plant response to abiotic stresses by regulating the expression of different genes $[13,23,60]$. Among HSF genes in plants, HsfA1 (a major transcriptional activator) is necessary to evoke the HSR. Indeed, the HsfA1-knockout mutant shows reduced expression of many HS-induced genes [45, 61]. Moreover, $H s f A 1 a$ is associated with Cd tolerance by improving melatonin biosynthesis [8]. In this study, RsHSF genes were significantly differentially expressed in response to various stresses. RsHSFA1s (RsHSF-18 and RsHSF-25) showed converse expression levels upon $24 \mathrm{~h}$ HS or $200 \mathrm{mg} / \mathrm{L} \mathrm{Pb}$, which was significantly down-regulated under $\mathrm{Pb}$ stress, indicating that the expression of HSFA1 may be repressed under $200 \mathrm{mg} / \mathrm{L} \mathrm{Pb}$ stress. In addition, RsHSF-18 and RsHSF-25 were differentially expressed under $50 \mathrm{mg} / \mathrm{L} \mathrm{Cd}$ stress, which may be related to the specific function in the $\mathrm{Cd}$ stress response. HsfA4A enhances $\mathrm{Cd}$ tolerance in wheat and rice and salt protection in Arabidopsis [62]. RsHSFA4s (RsHSF-08, RsHSF-16, RsHSF-17 and RsHSF22) were up-regulated, while only RsHSF-16 was highly expressed upon salt and $\mathrm{Cd}$ treatment. These upregulated genes might play specific roles in coping with stresses in radish plants. Unlike HSFAs, a considerable number of HSFBs and HSFCs are not reported to act as transcription activators, although tomato HSFB1 apparently plays a role as a transcriptional co-activator of HsfA1 under HS [17, 44, 63]. In this study, the expression of RsHSFBs was distinct. For example, RsHSF-08 and RsHSF18 had high expression level while RsHSF-11 and RsHSF33 were down-regulated upon $24 \mathrm{~h}$ HS. Intriguingly, the expression level of RsHSF-29 (an HSFC), was downregulated under all abiotic stresses except $200 \mathrm{mg} / \mathrm{L} \mathrm{Pb}$ treatment. HSFA1d and HSFA3 were significantly upregulated in radish under $\mathrm{HS}$ [30], while several HSFs, including HSFC1 and HSFB2a, were notably up-regulated under salt stress [64]. Some heavy metal stress responsive signaling molecules are activated on detecting $\mathrm{Cd}^{2+}$, such as calcium-dependent protein kinases (CDPKs) and mitogen-activated protein kinase (MAPKs), both of which consequently regulate various metal-responsive TF families (eg., NACs and HSFs) in radish nucleus [65]. Moreover, four radish HSF genes were up-regulated under $\mathrm{Cr}$ stress in radish [66]. Taken together, several RsHSF genes were differentially expressed upon abiotic stresses including heat, salt or heavy metal stress, and these results indicate that they might be involved in the plant response to abiotic stresses.
RsHSF gene expression during different stages and in tissues is associated with abiotic stresses tolerance. In this study, RsHSF genes exhibited tissue-specific expression patterns. At the seedling stage, RsHSF-04 and RsHSF-33 were lowly expressed in 20 and 14 DAS root, respectively. Throughout development stages, RsHSF-10 was only detected in 7 and 14. DAS root. AtHSF-16 (At4g36990) is a direct target gene of AtHsfA1s, which are critical for plants to initiate positive activities when coping with HS $[16$, 67]. RsHSF-03 is highly similar to AtHSF-16 (At4g36990) and exhibited high expression level during seedling and taproot-thickening stages. Notably, RsHSF19, the orthologous gene of AtHSF-02(At5g1682), was not expressed during all stages. These findings imply that the highly expressed genes among different stages and tissues may contribute to enhance stress tolerance in radish. Further characterization of these differentially expressed RsHSF genes would facilitate investigating the regulatory networks of abiotic stress responses in radish.

\section{Conclusions}

In summary, a total of 33 RsHSF genes were identified at the genome-wide level in radish. The protein motifs in most HSF members within one class were similar, and most HSF genes had only one intron. The expression patterns of several RsHSF genes were more similar within the same class than that among different classes. Moreover, comparative analysis revealed a series of paralogous RsHSF genes in radish and orthologous RsHSF genes in Arabidopsis and rice. These results may enhance our understanding of RsHSF functions and their involvement in the radish stress responses. Identification of RsHSF genes provides a rich resource for comprehensive investigation of the roles of HSF genes in regulatory networks of abiotic stress responses in plants. These findings will facilitate further functional characterization of RsHSF genes, and provide valuable information to clarify the molecular mechanism underlying abiotic stress responses in radish.

\section{Methods}

\section{Sequence collection and HSF identification}

Whole genome sequences of the radish HSF family were identified from the NODAI Radish genome database [31]. The HSF family sequences identified from Arabidopsis and Chinese cabbage were downloaded from the Arabidopsis Information Resource [37] and Brassica database (BRAD) [33], respectively. The HSF family members for rice (O. sativa) and grape ( $V$. vinifera) were obtained from the Rice Genome Annotation Project [36] and Grape Genome Browser (http://www.genoscope.cns. fr/externe/GenomeBrowser/Vitis/) [34], respectively.

To confirm the radish HSF family candidates, proteins with the HSF-type DBD domain (Pfam accession 
number: PF00447) were searched against the genome protein sequences using the HMM search tool with an E-value cut-off of 0.01 [32].

\section{Phylogenetic analysis and characterization of RsHSFs}

RsHSF DBD and oligomerization (HR-A/B or OD) domains were detected using SMART (http://smart.embl-heidelberg. de/) [68], Pfam [69], MARCOIL (http://toolkit.tuebingen. mpg.de/marcoil) and Heatster online tools (http://www. cibiv.at/services/hsf/info) [49]. NLS and NES domains were predicted using NLStradamus (http://www.moseslab.csb. utoronto.ca/NLStradamus/) and NESs (http://www.cbs.dtu. $\mathrm{dk} /$ services/NetNES/), respectively. RsHSF protein properties including molecular weight, theoretical $\mathrm{pI}$ and instability index were analyzed with the ExPASy ProtParam tool (https://www.expasy.org/). The alignment was firstly performed using MUSCLE and consequently the bestfit model was determined by MEGA X. The phylogenetic tree was constructed with specific parameters (model: JTT $+\mathrm{G}$ ) by IQ-TREE (1000 ultrafast bootstraps) using the maximum likelihood method [38]. The phylogenetic tree was displayed using EVOLVIEW (http://www.evolgenius.info/evolview/).

\section{Gene structure and conserved motif analysis}

RsHSF gene exton/intron organization was obtained from GSDS (http://gsds.cbi.pku.edu.cn/) [70] by aligning coding sequences with their corresponding genomic DNA sequences. The conserved motifs within the determined HSF groups were identified by MEME (version4.11.1) (http://meme-suite.org/) based on the following parameters: number of repetitions, any; maximum number of motifs, 25; and the optimum motif widths, 6-50 AAs [71]. The conserved motifs were visualized by Tbtools [72].

\section{Chromosomal localization and identification of orthologous and paralogous HSFs}

RsHSF gene sequences in scaffolds were obtained from the NODAI Radish Genome Database [31], and local BLAST was performed against Radish Genome Database [73]. Gene sequences with $\geq 98 \%$ identity and length difference $\leq 5$ base pairs were considered to be the same genes between two genomes, and the corresponding location of RsHSF genes in chromosomes was localized using MapChart Software [74]. The Multiple Collinearity Scan toolkit (MCScanX) was used to identify the RsHSF duplication events $[41,73]$. BLASTP was performed to identify the intra-species paralogous pairs using protein sequences with the following parameters settings: 1) alignment significance: E_VALUE (default: 1e-05); 2) MATCH_SCORE: final score (default: 50); 3) MATCH SIZE: number of genes required to call a collinear block (default: 5) and the maximum gaps (default: 25).
The OrthoMCL pipeline [43] was used with standard settings to identify potential orthologous and paralogous HSF genes in radish, Arabidopsis, and rice. The relationships of orthologous and paralogous genes among the three species were plotted using Circos software [75].

\section{Expression analyses of RsHSF genes by RNA-Seq}

To analyze the expression patterns of the RsHSF genes, the Illumina RNA-Seq data from five tissues (cortex, cambium, xylem, root tip and leaf) and six stages [7, 14, 20, 40, 60 and 90 days after sowing (DAS)] were collected from the radish reference genome (http://www.nodai-genome-d.org/) [31] (Additional file 11: Table S12). The expression level for each RsHSF gene was presented with the RPKM method and heat map was generated using TBtools [72].

\section{Abiotic stress treatments}

'NAU-YH' seeds, a radish advanced inbred line with small red global roots, were sterilised, rinsed and incubated for 3 days. Germinated seeds were transferred into a plug tray and cultured in a growth chamber at $25^{\circ} \mathrm{C}$ day $/ 18^{\circ} \mathrm{C}$ night with $14 \mathrm{~h}$ light $/ 10 \mathrm{~h}$ dark, $60 \%$ humidity and $12,000 \mathrm{~lx}$ light. Three weeks later, seedlings with $6-7$ true leaves at the cortex split stage were exposed to $38^{\circ} \mathrm{C}$ for 0,6 or 24 h (termed control, Heat6 or Heat24, respectively). For salt and heavy metal $(\mathrm{Cd}$ and $\mathrm{Pb})$ treatment, seedlings of identical sizes were grown in a plastic container with modified half-strength Hoagland nutrient solution as described previously [76]. The nutrient solution was changed every 3 days. One week later, the seedlings were treated with $\mathrm{CdCl}_{2} \cdot 2.5 \mathrm{H}_{2} \mathrm{O}(50 \mathrm{mg} / \mathrm{L}), \mathrm{Pb}\left(\mathrm{NO}_{3}\right)_{2}(100 \mathrm{mg} / \mathrm{L}$ or $200 \mathrm{mg} /$ L) and $\mathrm{NaCl}(150 \mathrm{mM}$ or $250 \mathrm{mM})$, respectively [29]. Seedlings grown in $\mathrm{Cd} / \mathrm{Pb}$-free nutrient solution were used as the control. In this study, three similar seedlings with 6 true leaves at the cortex split stage were used for each treatment, and each treatment was performed in triplicate. Additionally, $0.1 \mathrm{~g}$ root tissue was used for RNA isolation from each sample. These samples were immediately frozen in liquid nitrogen and stored at $-80^{\circ} \mathrm{C}$ for further analysis.

\section{RNA-Seq data and RT-qPCR analysis}

In this study, the raw RNA-Seq data was collected from NCBI Sequence Read Archive (SRA, http://www.ncbi. nlm.nih.gov/sra) using the SRA Toolkit (ver.2.8.2). After removing the adaptor sequences, contaminants and lowquality reads, the clean reads were mapped to the radish reference genome using TopHat2 [77]. Transcripts per Kilobase Million (TPM) values were estimated using Salmon [78] for different sample comparisons. Differential gene expression and the adjusted $p$ values were calculated using the edgeR package [79]. 
Total RNA was isolated from the control and treated radish roots using RNAsimple total RNA kit (Tiangen, Beijing, China).Then, the RNA was reverse transcribed into cDNA uisng PrimeScript ${ }^{\text {tw }}$ II 1st Strand cDNA Synthesis Kit (Takara, Dalian, China) according to the manufacturer's instructions. RT-qPCR analysis was conducted using SYBR Green PCR Master Mix (Takara, Dalian, China) [29]. RsActin was employed as the internal standard to normalize expression [80]. Each $20 \mu \mathrm{l}$ reaction contained $10 \mu \mathrm{l}$ of $2 \times$ SYBR Green PCR Master Mix (Takara, Dalian, China), $0.2 \mu \mathrm{M}$ of each primer and $2 \mu$ diluted cDNA. PCR was performed on an iCycler iQ real-time PCR detection system (BIO-RAD, USA) with the following thermal cycling conditions: $95^{\circ} \mathrm{C}$ for 3 min, and 40 cycles of $95^{\circ} \mathrm{C}$ for $5 \mathrm{~s}, 58{ }^{\circ} \mathrm{C}$ for $30 \mathrm{~s}$, and $72^{\circ} \mathrm{C}$ for $10 \mathrm{~s}$. The $2^{-\Delta \Delta C}$ T method was used to calculate relative expression level [81]. Three biological and technical replicates were performed. The significance of differences between groups was evaluated using a Student's $\mathrm{t}$ test. Analyses were performed with GraphPad Prism software (GraphPad Software, San Diego, California).

\section{Supplementary information}

Supplementary information accompanies this paper at https://doi.org/10. 1186/s12864-019-6121-3.

Additional file 1: Table S1. Amino acid sequences of the HSF proteins from NODAI Radish genome database. Table S2. CDS sequences of the HSF genes from NODAl Radish genome database. Table S3. DNA sequences of the HSF genes from NODAI Radish genome database.

Additional file 2: Figure S1 Protein sequence alignment of the DBD domain identified in all RsHsf genes. Figure S2. The LOGO of 25 amino acid motifs in HSF proteins. Figure S3. The networks of HSF genes in radish and Arabidopsis. This interrelation network has been constructed using radish and Arabidopsis orthologous gene pairs. Figure S4. The differential expression profiles of some HSF genes in radish under the Heat, salt, $\mathrm{Pb}$ and $\mathrm{Cd}$ treatments. The color scale of heatmap is based on the $\log _{2}$ Foldchange (a) and $\log _{2}$ TPM value (b), respectively.

Additional file 3: Table S4. Summary information of the RsHSF proteins.

Additional file 4: Table S5. HSFs in Arabidopsis, rice, and grape. Additional file 5: Table S6. Summary information of chromosomal location of RsHSFs in radish.

Additional file 6: Table S7. The different origins of HSF genes in radish. Additional file 7: Table S8. Duplicated genes of HSF in radish. Additional file 8: Table S9. The orthologous and paralogous gene pairs of HSF proteins among the radish, Arabidopsis and rice.

Additional file 9: Table S10. Tissue-specific expression of radish HSF genes.

Additional file 10: Table S11. Relative expression of RsHSF genes under different treatments determined by RT-qPCR.

Additional file 11: Table S12. The accession number of RNAseq data.

\section{Abbreviations}

CDS: Coding sequence; DAS: Days after sowing; DBD: DNA-binding domain; DEGs: Differentially expressed genes; HMM: Hidden Markov Model; HR-A/ B: Hydrophobic amino acid residues; HSF: Heat shock transcription factor; HSPs: Heat shock proteins; HSR: Heat stress response; MWs: Molecular weights; NES: Nuclear export signal; NLS: Nuclear localization Signal; OD: Oligomerization domain; pl: Theoretical isoelectric points; RTqPCR: Reverse-transcription quantitative PCR; TDR: Temperature-dependent repression; TF: Transcription factor; WGD: Whole-genome duplication; WGT: Whole genome triplication

\section{Acknowledgments}

Not applicable.

\section{Authors' contributions}

MT performed the data analysis and wrote the manuscript. LX and YW contributed powerful analytical tools. WC and YX performed the RNA extractions. $X L$ and $L F$ helped with the revise of manuscript. $L L$ conceived and designed the research. All authors read and approved the final manuscript.

\section{Funding}

This work was in part supported by grants from the Natural Science Foundation of China (31601766), National Key Technology R\&D Program of China (2017YFD0101803/06), Key Technology R\&D Program of Jiangsu Province (BE2016379), Jiangsu Agricultural S\&T Innovation Fund [CX (18)3067, CX(19)3032], Jiangsu Agricultural Industry Technology System (JATS2019), China Postdoctoral Science Foundation (2016T90472, 2015M570458) and Jiangsu Postdoctoral Science Foundation (1402021B). The funding bodies had no role in the design of the study, data analysis and interpretation, and preparation of the manuscript.

\section{Availability of data and materials}

The datasets supporting the conclusions of this article are included within the article and its additional files (Additional file 11: Table S12).

\section{Ethics approval and consent to participate}

In this study, all the radish lines were collected from germplasm bank of National Key Laboratory of Crop Genetics and Germplasm Enhancement, Nanjing Agricultural University. Collection of plant materials complied with the institutional, national and international provisions.

\section{Consent for publication}

Not applicable.

\section{Competing interests}

The authors declare that they have no competing interests.

Received: 19 May 2019 Accepted: 20 September 2019

Published online: 24 October 2019

\section{References}

1. Tester M, Bacic A. Abiotic stress tolerance in Grasses.From model plants to crop plants. Plant Physiol. 2005;137:791-3.

2. Yokotani N, Ichikawa T, Kondou Y, Iwabuchi M, Matsui M, Hirochika H, Oda K. Role of the rice transcription factor JAmyb in abiotic stress response. J Plant Res. 2013;126:131-9.

3. Mickelbart MV, Hasegawa PM, Bailey-Serres J. Genetic mechanisms of abiotic stress tolerance that translate to crop yield stability. Nat Rev Genet. 2015; 16(4):237-51.

4. Wang $R H$, Xu L, Zhu XW, Zhai LL, Wang Y, Yu RG, Gong YQ, Limera C, Liu LW. Transcriptome-wide characterization of novel and heatstress-responsive microRNAs in radish (Raphanus sativus L.) using next-generation sequencing. Plant Mol Biol Rep. 2015;33(4):867-80.

5. Lobell DB, Schlenker W, Costa-Roberts J. Climate trends and global crop production since 1980. Science. 2011;333(6042):616-20.

6. Jung KH, Ko HJ, Nguyen MX, Kim SR, Ronald P, An G. Genome-wide identification and analysis of early heat stress responsive genes in rice. J Plant Biol. 2012;55(6):458-68.

7. Wang PF, Song H, Li CS, Li AQ, Guan HS, Hou L, Wang XJ. Genome-wide dissection of the heat shock transcription factor family genes in Arachis. Front Plant Sci. 2017:8:106.

8. Cai SY, Zhang Y, Xu YP, Qi ZY, Li MQ, Ahammed GJ, Xia XJ, Shi K, Zhou HY, Reiter RJ, Yu JQ, Zhou J. HsfAla upregulates melatonin biosynthesis to confer cadmium tolerance in tomato plants. J Pineal Res. 2017;62(2):e12387. 
9. Scharf KD, Berberich T, Ebersberger I, Nover L. The plant heat stress transcription factor ( $\mathrm{Hsf}$ ) family: structure, function and evolution. BBA-Gene Regul Mec. 2012;1819(2):104-19.

10. Hossain Z, Nouri MZ, Komatsu S. Plant cell organelle proteomics in response to abiotic stress. J Proteome Res. 2012;11(1):37-48.

11. Singh R, Jwa NS. Understanding the responses of rice to environmental stress using proteomics. J Proteome Res. 2013;12(11):4652-69.

12. Nakashima K, Ito Y, Yamaguchi-Shinozaki K. Transcriptional regulatory networks in response to abiotic stresses in Arabidopsis and grasses. Plant Physiol. 2009;149(1):88-95.

13. Huang Y, Li MY, Wang F, Xu ZS, Huang W, Wang GL, Ma J, Xiong AS. Heat shock factors in carrot: genome-wide identification, classification, and expression profiles response to abiotic stress. Mol Biol Rep. 2015;42(5):893.

14. Todaka D, Nakashima K, Shinozaki K, Yamaguchi-Shinozaki K. Toward understanding transcriptional regulatory networks in abiotic stress responses and tolerance in rice. Rice. 2012;5(1):6.

15. Huang YC, Niu CY, Yang CR, Jinn TL. The heat stress factor HSFA6b connects ABA signaling and ABA-mediated heat responses. Plant Physiol. 2016;172(2):1182-99.

16. Ohama N, Kusakabe K, Mizoi J, Zhao H, Kidokoro S, Koizumi S, Takahashi F, Ishida T, Yanagisawa S, Shinozaki K, Yamaguchi-Shinozaki K. The transcriptional cascade in the heat stress response of Arabidopsis is strictly regulated at the level of transcription factor expression. Plant Cell. 2016;28(1):181-201.

17. Koskull-Döring PV, Scharf KD, Nover L. The diversity of plant heat stress transcription factors. Trends Plant Sci. 2007;12(10):452-7.

18. Björk JK, Sistonen L. Regulation of the members of the mammalian heat shock factor family. FEBS J. 2010;277(20):4126-39.

19. Cicero MP, Hubl ST, Harrison CJ, Littlefield O, Hardy JA, Nelson HCM. The wing in yeast heat shock transcription factor (HSF) DNA-binding domain is required for full activity. Nucleic Acids Res. 2001;29(8):1715-23.

20. Baniwal SK, Bharti K, Chan KY, Fauth M, Ganguli A, Kotak S, Mishra SK, et al. Heat stress response in plants: a complex game with chaperones and more than twenty heat stress transcription factors. J Biosci. 2004;29(4):471-87.

21. Nover L, Bharti K, Doring P, Mishra SK, Ganguli A, Scharf KD. Arabidopsis and the heat stress transcription factor world: how many heat stress transcription factors do we need? Cell Stress Chaperons. 2001;6:177-89.

22. Yang ZF, Wang YF, Gao Y, Zhou Y, Zhang EY, Hu YY, Yuan Y, Linag GH, Xu CW. Adaptive evolution and divergent expression of heat stress transcription factors in grasses. BMC Evol Biol. 2014;14:147.

23. Song XM, Liu GF, Duan WK, Liu TK, Huang ZN, Ren J, Li Y, Hou XL. Genomewide identification, classification and expression analysis of the heat shock transcription factor family in Chinese cabbage. Mol Gen Genomics. 2014; 289(4):541-51.

24. Guo JK, Wu J, Ji Q, Wang C, Luo L, Yuan Y, Wang YH, Wang J. Genome-wide analysis of heat shock transcription factor families in rice and Arabidopsis. J Genet Genomics. 2008;35(2):105-18.

25. Chauhan $H$, Khurana N, Agarwal P, Khurana P. Heat shock factors in rice (Oryza sativa L.): genome-wide expression analysis during reproductive development and abiotic stress. Mol Gen Genomics. 2011;286(2):171-87.

26. Lin YX, Jiang HY, Chu ZX, Tang XL, Zhu SW, Cheng BJ. Genome-wide identification, classification and analysis of heat shock transcription factor family in maize. BMC Genomics. 2011;12(1):76.

27. Qiao X, Li M, Li LT, Yin H, Wu JY, Zhang SL. Genome-wide identification and comparative analysis of the heat shock transcription factor family in Chinese white pear (Pyrus bretschneideri) and five other Rosaceae species. BMC Plant Biol. 2015;15(1):12.

28. Wang FM, Dong Q, Jiang HY, Zhu SW, Chen BJ, Xiang Y. Genome-wide analysis of the heat shock transcription factors in Populus trichocarpa and Medicago truncatula. Mol Biol Rep. 2012;39(2):1877-86.

29. Xu L, Wang Y, Liu W, Wang J, Zhu XW, Zhang KY, Yu RG, Wang RH, Xie Y, Zhang W, Gong YQ, Liu LW. De novo sequencing of root transcriptome reveals complex cadmium-responsive regulatory networks in radish (Raphanus sativus L.). Plant Sci. 2015;236:313-23.

30. Wang RH, Mei Y, Xu L, Zhu XW, Wang Y, Guo J, Liu LW. Genome-wide characterization of differentially expressed genes provides insights into regulatory network of heat stress response in radish (Raphanus sativus L.). Funct integr Genomics. 2018;18(2):225-39.

31. Mitsui $Y$, Shimomura M, Komatsu K, Namiki N, Shibata-Hatta M, Imai M, Katayose $Y$, Mukai $Y$, Kanamori H, Kurita K, Kagami T, Wakatsuki A, Ohyanagi H, Ikawa H, Minaka N, Nakagawa K, Shiwa Y, Sasaki T. The radish genome and comprehensive gene expression profile of tuberous root formation and development. Sci Rep. 2015;5:10835.
32. Finn RD, Clements J, Eddy SR. HMMER web server: interactive sequence similarity searching. Nucleic Acids Res. 2011;39(suppl_2):W29-37.

33. Cheng F, Liu SY, Wu J, Fang L, Sun SL, Liu B, Li PX, Hua W, Wang XW. BRAD, the genetics and genomics database for Brassica plants. BMC Plant Biol. 2011;11:136.

34. Liu GT, Chai FM, Wang Y, Jiang JZ, Duan W, Wang YT, Wang FF, Li SH, Wang LJ. Genome-wide identification and classification of HSF family in grape, and their transcriptional analysis under heat acclimation and heat stress. Hortic Plant J. 2018;4(4):133-43.

35. Goodstein DM, Shu S, Howson R, Neupane R, Hayes RD, Fazo J, Mitros T, Dirks W, Hellsten U, Putnam N, Rokhsar DS. Phytozome: a comparative platform for green plant genomics. Nucleic Acids Res. 2011;40(D1):D1178-86.

36. Kawahara Y, de la Bastide M, Hamilton JP, Kanamori H, McCombie WR, Ouyang S, Schwartz DC, Tanaka T, Wu JZ, Zhou SG, Childs KL, Davidson RM, Lin HN, Quesada-Ocampo L, Vaillancourt B, Sakai H, Lee SS, Kim J, Numa H, Itoh T, Buell CR, Matsumoto T. Improvement of the Oryza sativa Nipponbare reference genome using next generation sequence and optical map data. Rice. 2013;6(1):4.

37. Berardini TZ, Reiser L, Li D, Mezheritsky Y, Muller R, Strait E, Huala E. The Arabidopsis information resource: Making and mining the "gold standard" annotated reference plant genome. Genesis. 2015;53(8):474-85.

38. Nguyen LT, Schmidt HA, von Haeseler A, Minh BQ. IQ-TREE: a fast and effective stochastic algorithm for estimating maximum-likelihood phylogenies. Mol Biol Evol. 2014;32(1):268-74.

39. Wang $X$, Wang H, Wang J, Sun R, Wu J, Liu S, Bai Y, Mun JH, Bancroft I, Cheng $F$. The genome of the mesopolyploid crop species Brassica rapa. Nat Genet. 2011;43:1035-9.

40. Cannon SB, Mitra A, Baumgarten A, Young ND, May G. The roles of segmental and tandem gene duplication in the evolution of large gene families in Arabidopsis thaliana. BMC Plant Biol. 2004:4:10.

41. Wang Y, Tang H, Debarry JD, Tan X, Li J, Wang X, Lee TH, Jin H, Marler B, Guo H, Kissinger JC, Paterson AH. MCScanX: a toolkit for detection and evolutionary analysis of gene synteny and collinearity. Nucleic Acids Res. 2012;40(7):e49.

42. Moghe GD, Hufnagel DE, Tang HB, Xiao YL, Dworkin I, Town CD, Conner JK, Shiu SH. Consequences of whole-genome triplication as revealed by comparative genomic analyses of the wild radish Raphanus raphanistrum and three other Brassicaceae species. Plant Cell. 2014;26(5):1925-37.

43. Li L, Stoeckert CJ, Roos DS. OrthoMCL: identification of ortholog groups for eukaryotic genomes. Genome Res. 2003;13:2178-89.

44. Ohama N, Sato H, Shinozaki K, Yamaguchi-Shinozaki K. Transcriptional regulatory network of plant heat stress response. Trends Plant Sci. 2017; 22(1):53-65.

45. Yoshida T, Ohama N, Nakajima J, Kidokoro S, Mizoi J, Nakashima K, Maruyama K, Kim JM, Seki M, Todaka D, Osakabe Y, Sakuma Y, Schöffl F, Shinozaki K, Yamaguchi-Shinozaki K. Arabidopsis HsfAl transcription factors function as the main positive regulators in heat shock-responsive gene expression. Mol Gen Genomics. 2011;286(5-6):321-32.

46. Nishizawa-Yokoi A, Nosaka R, Hayashi H, Tainaka H, Maruta T, Tamoi M, Ikeda M, Ohme-Takagi M, Yoshimura K, Yabuta Y, Shigeoka S. HsfA1d and HsfA1e involved in the transcriptional regulation of HsfA2 function as key regulators for the Hsf signaling network in response to environmental stress. Plant Cell Physiol. 2011;52(5):933-45.

47. Heerklotz D, Döring P, Bonzelius F, Winkelhaus S, Nover L. The balance of nuclear import and export determines the intracellular distribution and function of tomato heat stress transcription factor HsfA2. Mol Cell Biol. 2001; 21(5):1759-68.

48. Kotak S, Port M, Ganguli A, Bicker F, Koskull-Döring PV. Characterization of C-terminal domains of Arabidopsis heat stress transcription factors (Hsfs) and identification of a new signature combination of plant class A Hsfs with AHA and NES motifs essential for activator function and intracellular localization. Plant J. 2004;39(1):98-112.

49. Berz J, Simm S, Schuster S, Scharf KD, Schleiff E, Ebersberger I. HEATSTER: a database and web server for identification and classification of heat stress transcription factors in plants. Bioinform Biol Insights. 2019;13: 1177932218821365.

50. Wendel JF. Genome evolution in polyploids. In: Doyle J, Gaut B, editors. Plant molecular evolution. Dordrecht: Springer; 2000. p. 225-49.

51. Bowers JE, Chapman BA, Rong J, Paterson AH. Unravelling angiosperm genome evolution by phylogenetic analysis of chromosomal duplication events. Nature. 2003;422:433-8. 
52. Beilstein MA, Nagalingum NS, Clements MD, Manchester SR, Mathews S. Dated molecular phylogenies indicate a Miocene origin for Arabidopsis thaliana. Proc Natl Acad Sci U S A. 2010;107:18724-8.

53. Nie SS, Zhang MJ, Zhang LG. Genome-wide identification and expression analysis of calmodulin-like (CML) genes in Chinese cabbage (Brassica rapa $\mathrm{L}$. ssp. pekinensis). BMC Genomics. 2017:18(1):842.

54. Wang W, Jiang W, Liu J, Li Y, Gai JY, Li Y. Genome-wide characterization of the aldehyde dehydrogenase gene superfamily in soybean and its potential role in drought stress response. BMC Genomics. 2017;18(1):518.

55. Li C, Wang Y, Xu L, Nie SS, Chen YL, Liang DY, Sun XC, Karanja BK, Luo XB, Liu LW. Genome-wide characterization of the MADS-box gene family in radish (Raphanus sativus L.) and assessment of its roles in flowering and floral organogenesis. Front Plant Sci. 2016;7:1390.

56. Ma J, Wang F, Li MY, Jiang Q, Tan GF, Xiong AS. Genome wide analysis of the NAC transcription factor family in Chinese cabbage to elucidate responses to temperature stress. Sci Hortic. 2014;165:82-90.

57. Song XM, Huang ZN, Duan WK, Ren J, Liu TK, Li Y, Hou XL. Genome-wide analysis of the bHIH transcription factor family in Chinese cabbage (Brassica rapa ssp. pekinensis). Mol Gen Genomics. 2014;289(1):77-91.

58. Yang YW, Tai PY, Chen Y, Li WH. A study of the phylogeny of Brassica rapa, B. nigra, Raphanus sativus, and their related genera using noncoding regions of chloroplast DNA. Mol Phylogenet Evol. 2002;23(2):268-75.

59. Lysak MA, Koch MA, Pecinka A, Schubert I. Chromosome triplication found across the tribe Brassiceae. Genome Res. 2005;15:516-25.

60. Hu WH, Hu GC, Han B. Genome-wide survey and expression profiling of heat shock proteins and heat shock factors revealed overlapped and stress specific response under abiotic stresses in rice. Plant Sci. 2009:176(4):583-90.

61. Liu HC, Liao HT, Charng YY. The role of class A1 heat shock factors (HSFA1s) in response to heat and other stresses in Arabidopsis. Plant Cell Environ. 2011;34(5):738

62. Pérez-Salamó I, Papdi C, Rigó G, Zsigmond L, Vilela B, Lumbreras V, Nagy I, Horváth B, Domoki M, Darula Z, Medzihradszky K, Bögre L, Koncz C, Szabados $\mathrm{L}$. The heat shock factor A4A confers salt tolerance and is regulated by oxidative stress and the mitogen-activated protein kinases MPK3 and MPK6. Plant Physiol. 2014;165(1):319-34.

63. Czarnecka-Verner E, Pan S, Salem T, Gurley WB. Plant class B HSFs inhibit transcription and exhibit affinity for TFIIB and TBP. Plant Mol Biol. 2004;56(1): $57-75$.

64. Sun $X C$, Xu L, Wang $Y$, Luo XB, Zhu XW, Kinuthia KB, Nie SS, Feng HY, Li C, Liu LW. Transcriptome-based gene expression profiling identifies differentially expressed genes critical for salt stress response in radish (Raphanus sativus L.). Plant Cell Rep. 2016;35(2):329-46.

65. Xu L, Wang Y, Zhang F, Tang MJ, Chen YL, Wang J, Karanja BK, Luo XB, Zhang W, Liu LW. Dissecting root proteome changes reveals new insight into cadmium stress response in radish (Raphanus sativus L.). Plant Cell Physiol. 2017:58(11):1901-13.

66. Xie Y, Ye S, Wang Y, Xu L, Zhu XW, Yang JL, Feng HY, Yu RG, Karanja B, Gong YQ, Liu LW. Transcriptome-based gene profiling provides novel insights into the characteristics of radish root response to $\mathrm{Cr}$ stress with next-generation sequencing. Front Plant Sci. 2015;6:202.

67. Charng YY, Liu HC, Liu NY, Chi WT, Wang CN, Chang SH, Wang TT. A heatinducible transcription factor, $\mathrm{HsfA} 2$, is required for extension of acquired thermotolerance in Arabidopsis. Plant Physiol. 2007;143(1):251-62.

68. Letunic I, Doerks T, Bork P. SMART 7: recent updates to the protein domain annotation resource. Nucleic Acids Res. 2012;40:D302-5.

69. Finn RD, Mistry J, Tate J, Coggill P, Heger A, Pollington JE, Gavin OL, Gunasekaran P, Ceric G, Forslund K, Holm L, Sonnhammer ELL, Eddy SR, Bateman A. The Pfam protein families database. Nucleic Acids Res. 2010;38 Database:211-22.

70. Hu B, Jin JP, Guo AY, Zhang H, Luo JC, Gao G. GSDS 2.0: an upgraded gene feature visualization server. Bioinformatics. 2015;31(8):1296-7.

71. Bailey $\mathrm{TL}$, Elkan C. The value of prior knowledge in discovering motifs with MEME. Proc Int Conf Intell Syst Mol Biol. 2005;3:21-9.

72. Chen CJ, Xia R, Chen H, He YH. TBtools, a toolkit for biologists integrating various biological data handling tools with a user-friendly interface. Preprint at https://doi.org/10.1101/289660 (2018).

73. Jeong YM, Kim N, Ahn BO, Oh M, Chung WH, Chung $H$, et al. Elucidating the triplicated ancestral genome structure of radish based on chromosomelevel comparison with the Brassica genomes. Theor Appl Genet. 2016;129(7): 1357-72.
74. Voorrips RE. MapChart: software for the graphical presentation of linkage maps and QTLs. J Hered. 2002;93(1):77-8.

75. Krzywinski M, Schein J, Birol I, Connors J, Gascoyne R, Horsman D, Jones SJ, Marra MA. Circos: an information aesthetic for comparative genomics. Genome Res. 2009;19:1639-45.

76. Xu L, Wang Y, Zhai LL, Xu Y, Wang LJ, Zhu XW, Gong YQ, Yu RG, Limera C, Liu LW. Genome-wide identification and characterization of cadmiumresponsive microRNAs and their target genes in radish (Raphanus sativus L.) roots. J Exp Bot. 2013;64:4271-87.

77. Kim D, Pertea G, Trapnell C, Pimentel H, Ryan Kelley R, Salzberg SL. TopHat2: accurate alignment of transcriptomes in the presence of insertions, deletions and gene fusions. Genome Biol. 2013;14:R36.

78. Patro R, Duggal G, Love MI, Irizarry RA, Kingsford C. Salmon provides fast and bias-aware quantification of transcript expression. Nat Meth. 2017;14(4):417-9.

79. Robinson MD, McCarthy DJ, Smyth GK. EdgeR: a bioconductor package for differential expression analysis of digital gene expression data. Bioinformatics. 2010;26(1):139-40.

80. Xu YY, Zhu XW, Gong YQ, Xu L, Wang Y, Liu LW. Evaluation of reference genes for gene expression studies in radish (Raphanus sativus L.) using quantitative real-time PCR. Biochem Bioph Res Co. 2012;424(3):398-403.

81. Livak KJ, Schmittgen TD. Analysis of relative gene expression data using real-time quantitative PCR and the $2^{-\Delta \Delta C T}$ method. Methods. 2001:25:402-8.

\section{Publisher's Note}

Springer Nature remains neutral with regard to jurisdictional claims in published maps and institutional affiliations.
Ready to submit your research? Choose BMC and benefit from:

- fast, convenient online submission

- thorough peer review by experienced researchers in your field

- rapid publication on acceptance

- support for research data, including large and complex data types

- gold Open Access which fosters wider collaboration and increased citations

- maximum visibility for your research: over $100 \mathrm{M}$ website views per year

At BMC, research is always in progress.

Learn more biomedcentral.com/submissions 